01

\title{
Изменение нормальных координат и геометрических параметров молекулы в лазерном поле
}

\author{
(C) А.С. Корнев, К.И. Суворов, В.Е. Чернов, Б.А. Зон \\ Воронежский государственный университет, \\ 394006 Воронеж, Россия \\ ฯ e-mail: zon@niif.vsu.ru
}

Поступила в редакцию 21.08.2017 г.

Получены общие формулы, описывающие изменение равновесных расстояний, валентных углов и частот колебаний многоатомной молекулы в сильном лазерном поле. Показано, что во втором порядке по напряженности поля координаты нормальных колебаний молекулы не изменяются. Для нелинейной трехатомной молекулы типа $A_{2} B$ изменение геометрических характеристик и частот колебаний найдены в явном виде.

DOI: $10.21883 /$ OS.2018.01.45353.183-17

\section{Введение}

Взаимодействие лазерного излучения с многоатомными молекулами активно изучается как экспериментально, так и теоретически. Среди достижений последних лет можно указать исследование лазерного химического синтеза $[1,2]$, лазерный контроль движения молекулярных электронов $[3,4]$ и изменения оптических свойств газа когерентно вращающихся молекул [5-8], спектроскопию комбинационного [9] и когерентного антистоксова рассеяния света [10], перегруппировку атомов при лазерной фрагментации молекул [11], наблюдение двухэлектронного отрыва от анионов $\mathrm{SF}_{6}^{-}[12,13]$ и $\mathrm{F}^{-}\left(\mathrm{NF}_{3}\right)_{n}[14]$.

В недавнем эксперименте [15] была продемонстрирована важная роль „одетого полем“ атома при рассеянии на нем электрона в поле лазерного излучения (laser-assisted electron scattering, LAES), теоретически рассмотренного в работе [16]. Рассматриваемая в настоящей работе теория представляет собой вариант концепции „одетой полем“ многоатомной молекулы, в которой происходит индуцированное полем изменение геометрических и спектроскопических характеристик. Это изменение связано с тем, что вследствие поляризуемости молекулы ее энергия во внешнем поле изменяется. Поэтому равновесная конфигурация молекулы в поле, соответствующая минимуму потенциальной энергии, может отличаться от равновесной конфигурации свободной молекулы.

При рассмотрении этого эффекта мы используем метод, предложенный в [17] и развитый в [18] для расчета неадиабатических восприимчивостей молекул в постоянном поле. Основное отличие постоянного поля от переменного в данном случае состоит в том, что в постоянном поле для полярных молекул возникают поправки к энергии молекулы нечетных порядков по напряженности поля, которых нет в переменных полях ИК и оптических частот [19]. Следует также отметить неадиабатическое исследование оптических свойств простей- ших молекул, проведенное в работе [20] вариационным методом с использованием осцилляторных функций. Обзор дальнейших работ этих авторов, в частности, проявление изменений геометрических параметров и колебательных спектров молекул (иными словами, колебательного эффекта Штарка [21]) в эффекте Керра, а также в генерации второй (в электрическом поле) и третьей гармоник дан в работе [22].

Рассмотрение данного явления для двухатомных молекул было проведено в работе [23]. В этом простейшем случае поляризуемость молекулы приводит к изменению равновесного межъядерного расстояния и частот ядерных колебаний. Это, в свою очередь, приводит к изменению факторов Франка-Кондона, что проявляется в изменениях вероятностей электромагнитных переходов молекулы. Проведенные в работах [24-27] расчеты вероятностей туннельной ионизации ряда двухатомных молекул показали, что из-за указанного изменения факторов Франка-Кондона вероятность ионизации молекул изменяется до двух раз.

В многоатомных молекулах в отличие от двухатомных внешнее поле изменяет не только межъядерные расстояния и частоты колебаний ядер, но и валентные углы. Поскольку поляризуемость молекулы является тензорной величиной, изменение всех этих параметров зависит от ориентации молекулы относительно направления поля. Отметим, что взаимодействие лазерного излучения с пространственно-ориентированными молекулами исследуется экспериментально во многих работах (см., например, [28-31]).

Изменение геометрических параметров в многоатомных молекулах наблюдалось при изучении кулоновского взрыва (см., например, [32-40]). Такое изменение сопровождается в общем случае изменением нормальных координат, определяющих положения ядер атомов, входящих в состав молекулы. Как известно [41], лагранжиан молекулы, записанный в этих координатах, представляет собой сумму независимых осцилляторов, которые описывают колебательный спектр молекулы в гармониче- 
ском приближении. Одним из результатов настоящей работы является доказательство утверждения, что с точностью до квадратичных по полю членов нормальные координаты молекулы в поле не изменяются по сравнению с нормальными координатами свободной молекулы. С указанной точностью получены также простые аналитические выражения для новых равновесных значений нормальных координат и новых частот гармонических колебаний.

В следующем разделе рассмотрена общая теория изменения колебательных параметров многоатомной молекулы под воздействием монохроматического лазерного излучения. Краткое изложение этих результатов опубликовано в [42]. В разделе „Лазерная деформация нелинейной трехатомной молекулы $A_{2} B^{\text {“ }}$ в качестве примера выписаны явные формулы, описывающие деформацию симметричной нелинейной молекулы $A_{2} B$. Численные результаты для молекул $\mathrm{H}_{2} \mathrm{O}, \mathrm{H}_{2} \mathrm{O}^{+}$и $\mathrm{SO}_{2}$ приведены в разделе „Численные результаты“. В работе используется атомная система единиц $\left(\hbar=m_{e}=e=1\right)$.

\section{Общий формализм}

Рассмотрим изменение колебательных параметров многоатомной молекулы под воздействием внешнего электромагнитного поля. Обозначим нормальные координаты молекулы $\boldsymbol{Q}=\left\{Q_{1}, Q_{2}, \ldots, Q_{n}\right\}$, где $n$ - число колебательных степеней свободы молекулы. В нормальных координатах классический лагранжиан колебательного движения имеет вид суммы лагранжианов независимых гармонических осцилляторов [41]:

$$
\mathscr{L}_{0}=\frac{1}{2} \sum_{k=1}^{n} M_{k} \dot{Q}_{k}^{2}-\frac{1}{2} \sum_{k=1}^{n} M_{k} \omega_{0 k}^{2}\left(Q_{k}-Q_{e k}\right)^{2} .
$$

Здесь $\omega_{0 k}$ - частота $k$-й нормальной колебательной моды, $Q_{e k}$ - равновесное значение координаты $Q_{k}, M_{k}-$ приведенная масса, соответствующая $k$-й моде. Предполагается, что вырожденные колебания отсутствуют.

Изменение колебательных параметров во внешнем линейно поляризованном электрическом поле

$$
\mathbf{F}(t)=\mathbf{F}_{0} \cos \Omega t
$$

обусловлено трансформацией потенциальной поверхности молекулы вследствие штарковского сдвига энергии. С точностью до слагаемых $\sim F_{0}^{2}$ этот сдвиг определяется тензором динамической дипольной поляризуемости молекулы $\alpha_{i j}(\Omega)$ :

$$
\Delta E_{\mathrm{Stark}}=-\frac{1}{4} \alpha_{i j} F_{0 i} F_{0 j}
$$

В формуле (2) и всюду далее предполагается суммирование по повторяющимся индексам $i, j=1,2,3$. Поляризуемость $\alpha_{i j}$ зависит от нормальных координат $\left\{Q_{k}\right\}$. Поэтому с учетом (2) уравнение потенциальной поверхности молекулы после разложения в ряд Тейлора принимает вид:

$$
\begin{aligned}
\mathscr{U}(\boldsymbol{Q}) & \approx \frac{1}{2} \sum_{k} M_{k} \omega_{0 k}^{2}\left(Q_{k}-Q_{e k}\right)^{2} \\
& -\frac{1}{4}\left[\alpha_{i j}^{(0)}+\sum_{k} \alpha_{i j}^{(1 k)}\left(Q_{k}-Q_{e k}\right)\right. \\
& \left.+\frac{1}{2} \sum_{k k^{\prime}} \alpha_{i j}^{\left(2 k k^{\prime}\right)}\left(Q_{k}-Q_{e k}\right)\left(Q_{k^{\prime}}-Q_{e k^{\prime}}\right)\right] F_{0 i} F_{0 j}
\end{aligned}
$$

Здесь $\alpha_{i j}^{(0)}$ - поляризуемость при равновесных значениях нормальных координат молекулы, $\left\{Q_{e k}\right\}$,

$$
\alpha_{i j}^{(1 k)}=\left.\frac{\partial \alpha_{i j}}{\partial Q_{k}}\right|_{\boldsymbol{Q}=\boldsymbol{Q}_{e}}, \quad \alpha_{i j}^{\left(2 k k^{\prime}\right)}=\left.\frac{\partial^{2} \alpha_{i j}}{\partial Q_{k} \partial Q_{k^{\prime}}}\right|_{\boldsymbol{Q}=\boldsymbol{Q}_{e}} .
$$

Для потенциальной энергии (3) лагранжиан не является диагональной квадратичной формой в отличие от (1). Однако надлежащим сдвигом положений равновесия выражение (3) приводится к квадратичной форме, которая затем может быть диагонализована. Для этой цели выражение (3) удобно представить в матричной форме:

$$
\begin{aligned}
& \mathscr{U}(\boldsymbol{Q})=-\frac{1}{4} \alpha_{i j}^{(0)} F_{0 i} F_{0 j} \\
& +\frac{1}{2} \sum_{k k^{\prime}} V_{k k^{\prime}}\left(Q_{k}-Q_{e k}\right)\left(Q_{k^{\prime}}-Q_{e k^{\prime}}\right)-\sum_{k} b_{k}\left(Q_{k}-Q_{e k}\right) .
\end{aligned}
$$

Здесь

$$
\begin{gathered}
\left.V_{k k^{\prime}} \equiv \frac{\partial^{2} \mathscr{U}}{\partial Q_{k} \partial Q_{k^{\prime}}}\right|_{\boldsymbol{Q}_{=} \boldsymbol{Q}_{e}} \\
=\left[M_{k} \omega_{0 k}^{2}-\frac{1}{4} \alpha_{i j}^{\left(2 k k^{\prime}\right)} F_{0 i} F_{0 j}\right] \delta_{k k^{\prime}}-\frac{1}{4} \alpha_{i j}^{\left(2 k k^{\prime}\right)} F_{0 i} F_{0 j}\left(1-\delta_{k k^{\prime}}\right), \\
b_{k}=\frac{1}{4} \alpha_{i j}^{(1 k)} F_{0 i} F_{0 j} .
\end{gathered}
$$

При записи матрицы $\hat{V}$ в форме (5) в явном виде видна малость ее недиагональных элементов $\sim F_{0}^{2}$, которая будет использоваться в дальнейшем.

Минимальное значение потенциальной энергии (4) достигается при таких значениях нормальных координат $\left\{Q_{E l}\right\}$, которые, учитывая симметричность матрицы $\hat{V}$ $\left(V_{k k^{\prime}}=V_{k^{\prime} k}\right)$, определяются из уравнений

$$
\left.\frac{\partial \mathscr{U}}{\partial Q_{k}}\right|_{\boldsymbol{Q}_{=} \boldsymbol{Q}_{E}}=\sum_{k^{\prime}} V_{k k^{\prime}}\left(Q_{k^{\prime}}-Q_{e k^{\prime}}\right)-b_{k}=0 .
$$

Решение системы линейных неоднородных алгебраических уравнений (7) выражается в соответствии с правилом Крамера:

$$
Q_{E k}=Q_{e k}+\frac{\operatorname{det} \hat{W}^{(k)}}{\operatorname{det} \hat{V}} .
$$


Здесь матрица $\hat{W}^{(k)}$ получается из $\hat{V}$ заменой $k$-го столбца вектором $\boldsymbol{b}$. Предположение об устойчивом равновесии молекулы означает, что матрица $\hat{V}$ не является особой.

B $\operatorname{det} \hat{V}$ в (8) все недиагональные элементы $\sim F_{0}^{2}$. С этой точностью $\operatorname{det} \hat{V}$ равен произведению диагональных элементов:

$$
\operatorname{det} \hat{V} \approx \prod_{l=1}^{n} V_{l l}+o\left(F_{0}^{2}\right)
$$

Здесь символ $o\left(F_{0}^{2}\right)$ означает наличие величин четвертой и более высоких степеней $F_{0}$. Соотношение (9) легко проверяется для $n=2$ и может быть доказано для произвольных $n$. Для $\operatorname{det} \hat{W}^{(k)}$ с использованием (9) получаем

$$
\operatorname{det} \hat{W}^{(k)} \approx b_{k} \prod_{l \neq k} V_{l l}
$$

Поэтому, если штарковский сдвиг достаточно мал, то выражение (8) принимает вид

$$
Q_{E k} \approx Q_{e k}+\frac{\alpha_{i j}^{(1 k)} F_{0 i} F_{0 j}}{4 M_{k} \omega_{0 k}^{2}}
$$

Изменив начало координат в соответствии с (8) и учитывая симметричность матрицы $\hat{V}$, потенциальную энергию (4) можно представить в следующем виде:

$$
\begin{gathered}
\mathscr{U}(\boldsymbol{Q})=\mathscr{U}_{0}+\sum_{k}\left\{-b_{k}+\sum_{k^{\prime}} V_{k k^{\prime}}\left(Q_{E k^{\prime}}-Q_{e k^{\prime}}\right)\right\}\left(Q_{k}-Q_{E k}\right) \\
+\frac{1}{2} \sum_{k k^{\prime}} V_{k k^{\prime}}\left(Q_{k}-Q_{E k}\right)\left(Q_{k^{\prime}}-Q_{E k^{\prime}}\right), \\
\mathscr{U}_{0}=-\frac{1}{4} \alpha_{i j}^{(0)} F_{0 i} F_{0 j}+\frac{1}{2} \sum_{k k^{\prime}} V_{k k^{\prime}}\left(Q_{E k}-Q_{e k}\right)\left(Q_{E k^{\prime}}-Q_{e k^{\prime}}\right) \\
-\sum_{k} b_{k}\left(Q_{E k}-Q_{e k}\right) .
\end{gathered}
$$

Выражение в фигурных скобках в этой формуле обращается в нуль вследствие условия (7), поэтому

$$
\mathscr{U}(\boldsymbol{Q})=\mathscr{U}_{0}+\frac{1}{2} \sum_{k k^{\prime}} V_{k k^{\prime}}\left(Q_{k}-Q_{E k}\right)\left(Q_{k^{\prime}}-Q_{E k^{\prime}}\right) .
$$

Лагранжиан с потенциальной энергией (11) является недиагональной квадратичной формой:

$$
\mathscr{L}=\frac{1}{2} \sum_{k} M_{k} \dot{Q}_{k}^{2}-\frac{1}{2} \sum_{k k^{\prime}} V_{k k^{\prime}}\left(Q_{k}-Q_{E k}\right)\left(Q_{k^{\prime}}-Q_{E k^{\prime}}\right) .
$$

Соответствующая система уравнений Лагранжа

$$
M_{k} \ddot{Q}_{k}+\sum_{k^{\prime}} V_{k k^{\prime}}\left(Q_{k^{\prime}}-Q_{E k^{\prime}}\right)=0
$$

имеет решение

$$
Q_{k}-Q_{E k^{\prime}}=\xi_{0 k} e^{-i \omega t}
$$

с неизвестными константами $\left\{\xi_{0 k}\right\}$ и $\omega$. В результате уравнения (13) превращаются в систему линейных однородных алгебраических уравнений относительно амплитуд $\left\{\xi_{0 k}\right\}$, условие нетривиальной разрешимости которых приводит к секулярному уравнению:

$$
0=\operatorname{det}\left\|V_{k k^{\prime}}-M_{k} \omega^{2} \delta_{k k^{\prime}}\right\|=\prod_{k=1}^{n}\left(V_{k k}-M_{k} \omega^{2}\right)+o\left(F_{0}^{2}\right) \text {. }
$$

Второе равенство в (14) записано с точностью до $\sim F_{0}^{2}$ на основе формулы (9). Решения этого уравнения дают частоты нормальных колебаний, измененные лазерным полем. С учетом (5) они принимают значения

$$
\omega_{k}^{2} \approx \frac{V_{k k}}{M_{k}}=\omega_{0 k}^{2}-\frac{\alpha_{i j}^{(2 k k)}}{4 M_{k}} F_{0 i} F_{0 j} .
$$

Соотношения (10) и (15) решают поставленную задачу. Частоты колебаний в поле отличаются от частот свободной молекулы малыми поправками. Нормальные координаты в поле отличаются от нормальных координат свободной молекулы малыми сдвигами положений равновесия. Формулы (10) и (15) аналогичны полученным в [23] для двухатомных молекул и показывают, что при учете лишь квадратичного эффекта Штарка параметры нормальных колебаний многоатомных молекул изменяются независимо.

Приближения, сделанные в (14) и (15), допустимы при выполнении условия

$$
\left|\alpha_{i j}^{\left(2 k k^{\prime}\right)}\right| F_{0}^{2} \ll\left|M_{k} \omega_{0 k}^{2}-M_{k^{\prime}} \omega_{0 k^{\prime}}^{2}\right|,
$$

аналогичного условию применимости теории возмущений для изолированных квантовых уровней. При наличии вырождения, например в линейной молекуле, условие (16) нарушается. Это приводит к невозможности факторизации в (14). Если для определенности положить колебательные моды $k=1,2$ вырожденными $\left(\omega_{01}=\omega_{02}=\omega_{0}, M_{1}=M_{2}=M\right)$, то выражение (15) примет вид

$$
\begin{aligned}
\omega_{1,2}^{2} & =\omega_{0}^{2}-\frac{1}{8 M}\left\{\alpha_{i j}^{(211)}+\alpha_{i j}^{(222)}\right. \\
& \left. \pm\left[\left(\alpha_{i j}^{(211)}-\alpha_{i j}^{(222)}\right)^{2}+4 \alpha_{i j}^{(212) 2}\right]^{1 / 2}\right\} F_{0 i} F_{0 j}+o\left(F_{0}^{2}\right),
\end{aligned}
$$

что указывает на взаимодействие между взаимно вырожденными модами через величины $\alpha_{i j}^{(212)}$. Следствием является снятие вырождения.

В качестве примера здесь можно привести простейшую линейную симметричную трехатомную молекулу $\mathrm{CO}_{2}$ с четырьмя колебательными степенями свободы. Две из них (пусть их номера $k=1,2$ ) вырождены. Они соответствуют колебаниям валентного угла возле значения $180^{\circ}$ во взаимно перпендикулярных плоскостях, пересекающихся по оси молекулы. Под воздействием лазерного поля их частоты изменяются в соответствии c (17). Однако наличие центра инверсии в орбиталях молекулы $\mathrm{CO}_{2}$ приводит к равенству нулю первых 
производных поляризуемостей по этим нормальным координатам:

$$
\alpha_{i j}^{(11)}=\alpha_{i j}^{(12)}=0 .
$$

Поэтому положения равновесия в данных колебательных модах не изменяются. Действительно, при наличии центра инверсии нет физических причин для возникновения „излома“ линейной симметричной молекулы в монохроматическом лазерном поле. Аксиальная симметрия приводит к равноправию данных вырожденных мод, а значит, к равенству $\alpha_{i j}^{(211)}=\alpha_{i j}^{(222)}=\alpha_{i j}^{(212)}$. Поэтому выражение (17) упрощается:

$$
\begin{gathered}
\omega_{1}=\omega_{0}+o\left(F_{0}^{2}\right), \\
\omega_{2}^{2}=\omega_{0}^{2}-\frac{\alpha_{i j}^{(211) 2}}{2 M} F_{0 i} F_{0 j}+o\left(F_{0}^{2}\right) .
\end{gathered}
$$

\section{Лазерная деформация нелинейной трехатомной молекулы $A_{2} B$}

\section{Нормальные колебания}

Рассмотрим согласование нормальных координат с приведенными массами на примере симметричной нелинейной трехатомной молекулы $A_{2} B$ в отсутствие внешних полей. В анализе колебательного движения мы будем следовать [43], а также [44].

Пусть массы ядер будут равны соответственно $m_{A}$, $m_{B}$, длина связи $A-B$ в положении равновесия $l$, половина валентного угла $\gamma$. Поместим молекулу в декартову плоскость $y z$ симметрично относительно оси $z$ и совместим атом $B$ с началом координат. Деформацию молекулы в настоящей работе удобно задавать векторами смещений ее ядер $\boldsymbol{u}_{1,3}=\left\{y_{1,3}, z_{1,3}\right\}$ - для ядер $A, \boldsymbol{u}_{2}=\left\{y_{2}, z_{2}\right\}$ - для ядра $B$ (рис. 1). Смещения предполагаются малыми по сравнению с длиной связи. Отметим, что при любой деформации молекула остается плоской.

Отсутствие поступательного и вращательного движений молекулы как целого приводит к дополнительным условиям для координат:

$$
\begin{gathered}
m_{A}\left(y_{1}+y_{3}\right)+m_{B} y_{2}=0, \\
m_{A}\left(z_{1}+z_{3}\right)+m_{B} z_{2}=0, \\
\sin \gamma\left(z_{1}-z_{3}\right)-\cos \gamma\left(y_{1}+y_{3}\right)=0 .
\end{gathered}
$$

Удобно ввести обобщенные координаты симметрии:

$$
\begin{aligned}
& Q_{a}=y_{1}+y_{3}, \\
& Q_{s 1}=y_{1}-y_{3}, \\
& Q_{s 2}=z_{1}+z_{3} .
\end{aligned}
$$

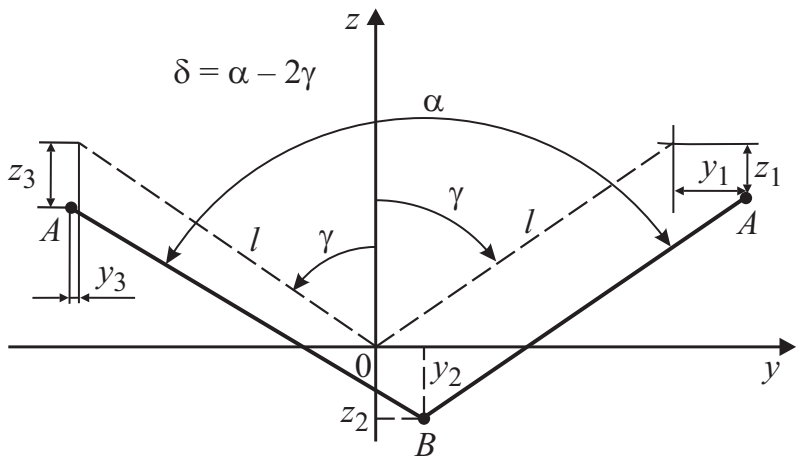

Рис. 1. Деформация молекулы $A_{2} B$ в декартовых координатах.

Тогда компоненты векторов $\boldsymbol{u}_{1,2,3}$ выражаются через координаты (20) с учетом (19) с помощью соотношений

$$
\begin{gathered}
y_{1}=\frac{1}{2}\left(Q_{a}+Q_{s 1}\right), \quad y_{2}=-\frac{m_{A}}{m_{B}} Q_{a}, \quad y_{3}=\frac{1}{2}\left(Q_{a}-Q_{s 1}\right), \\
z_{1}=\frac{1}{2}\left(Q_{s 2}+Q_{a} \cot \gamma\right), \quad z_{2}=-\frac{m_{A}}{m_{B}} Q_{s 2} \\
z_{3}=\frac{1}{2}\left(Q_{s 2}-Q_{a} \cot \gamma\right) .
\end{gathered}
$$

Потенциальную энергию целесообразно выбирать в рамках валентно-силовой модели в приближении гармонического осциллятора:

$$
\mathscr{U}=\frac{1}{2} k_{1}\left(q_{1}^{2}+q_{3}^{2}\right)+\frac{1}{2} k_{2} l^{2} \delta^{2} .
$$

Здесь $q_{1}=l_{12}-l, q_{3}=l_{32}-l-$ изменения длин связей вследствие деформации, $\delta=\alpha-2 \gamma-$ изменение валентного угла, $l_{12}, l_{32}$ и $\alpha$ - длины соответствующих связей и значение валентного угла в деформированной молекуле, $k_{1,2}$ - силовые постоянные.

Потенциальная энергия в координатах симметрии (20) имеет вид

$$
\begin{aligned}
\mathscr{U}= & \frac{1}{4} k_{1}\left(\frac{2 m_{A}}{m_{B}}+\frac{1}{\sin ^{2} \gamma}\right)\left(1+\frac{2 m_{A}}{m_{B}} \sin ^{2} \gamma\right) Q_{a}^{2} \\
& +\frac{1}{4}\left(k_{1} \sin ^{2} \gamma+2 k_{2} \cos ^{2} \gamma\right) q_{s 1}^{2} \\
& +\frac{M^{2}}{4 m_{B}^{2}}\left(k_{1} \cos ^{2} \gamma+2 k_{2} \sin ^{2} \gamma\right) q_{s 2}^{2} \\
& -\frac{M q_{s 1} q_{s 2}}{2 m_{B}}\left(2 k_{2}-k_{1}\right) \sin \gamma \cos \gamma,
\end{aligned}
$$

где $M=2 m_{A}+m_{B}$ - масса молекулы.

Функция Лагранжа с потенциальной энергией (23) принимает вид

$$
\mathscr{L}=\mathscr{L}_{a}+\mathscr{L}_{s}
$$

Здесь слагаемое

$$
\mathscr{L}_{a}=\frac{1}{2} M_{a} \dot{Q}_{a}^{2}+\frac{1}{2} M_{a} \omega_{a}^{2} Q_{a}^{2}
$$




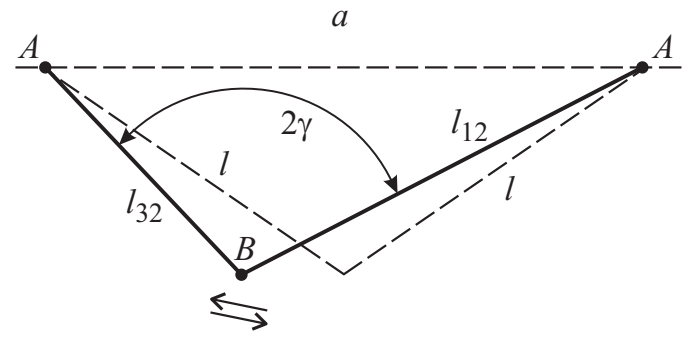

Рис. 2. Антисимметричное нормальное колебание молекулы $A_{2} B$.

соответствует антисимметричному движению (рис. 2). Оно подобно движению изолированного линейного гармонического осциллятора, имеющего массу

$$
M_{a}=\frac{1}{2} m_{A}\left(\frac{2 m_{A}}{m_{B}}+\frac{1}{\sin ^{2} \gamma}\right)
$$

и частоту

$$
\omega_{a}^{2}=\frac{k_{1}}{m_{A}}\left(1+\frac{2 m_{A}}{m_{B}} \sin ^{2} \gamma\right)
$$

Поэтому координата $Q_{a}$ является нормальной.

Второе слагаемое в (24)

$$
\begin{aligned}
\mathscr{L}_{s}= & \frac{1}{2} m_{A} \dot{Q}_{s 1}^{2}+\frac{1}{2} \frac{m_{A} M}{m_{B}} \dot{Q}_{s 2}^{2} \\
& -\frac{1}{2}\left(k_{1} \sin ^{2} \gamma+2 k_{2} \cos ^{2} \gamma\right) Q_{s 1}^{2} \\
& -\frac{M^{2}}{2 m_{B}^{2}}\left(k_{1} \cos ^{2} \gamma+2 k_{2} \sin ^{2} \gamma\right) Q_{s 2}^{2} \\
& +Q_{s 1} Q_{s 2} \frac{M}{m_{B}}\left(2 k_{2}-k_{1}\right) \sin \gamma \cos \gamma
\end{aligned}
$$

соответствует симметричному движению. Однако наличие недиагонального слагаемого $\sim Q_{s 1} Q_{s 2}$ в квадратичной форме (27) указывает на связь между степенями свободы $Q_{s 1}$ и $Q_{s 2}$. Поэтому данные обобщенные координаты не являются нормальными.

Переменные $Q_{s 1}$ и $Q_{s 2}$ удовлетворяют уравнениям Лагранжа:

$$
\begin{aligned}
& m_{A} \ddot{Q}_{s 1}+\left(k_{1} \sin ^{2} \gamma+2 k_{2} \cos ^{2} \gamma\right) Q_{s 1} \\
&- \frac{M}{m_{B}}\left(2 k_{2}-k_{1}\right) \sin \gamma \cos \gamma Q_{s 2}=0, \\
& m_{A} \ddot{Q}_{s 2}+\frac{M}{m_{B}}\left(k_{1} \cos ^{2} \gamma+2 k_{2} \sin ^{2} \gamma\right) Q_{s 2} \\
&-\left(2 k_{2}-k_{1}\right) \sin \gamma \cos \gamma Q_{s 1}=0 .
\end{aligned}
$$

Их решения ищутся в виде гармонических колебаний

$$
Q_{s 1}=a_{1} e^{-i \omega t}, \quad Q_{s 2}=a_{2} e^{-i \omega t}
$$

с неизвестными амплитудами $a_{1}, a_{2}$ и частотой $\omega$.

Подстановка (29) в (28) приводит к системе линейных алгебраических уравнений для амплитуд:

$$
\begin{aligned}
& {\left[-m_{A} \omega^{2}+\right.}\left.\left(k_{1} \sin ^{2} \gamma+2 k_{2} \cos ^{2} \gamma\right)\right] a_{1} \\
&- \frac{M}{m_{B}}\left(2 k_{2}-k_{1}\right) \sin \gamma \cos \gamma a_{2}=0, \\
& {\left[-m_{A} \omega^{2}+\frac{M}{m_{B}}\left(k_{1} \cos ^{2} \gamma+2 k_{2} \sin ^{2} \gamma\right)\right] a_{2} } \\
&-\left(2 k_{2}-k_{1}\right) \sin \gamma \cos \gamma a_{1}=0 .
\end{aligned}
$$

Условие нетривиальной разрешимости (30) приводит к секулярному уравнению для частот нормальных колебаний:

$$
\begin{aligned}
\omega^{4} & -\omega^{2}\left[\frac{k_{1}}{m_{A}}\left(1+\frac{2 m_{A}}{m_{B}} \cos ^{2} \gamma\right)\right. \\
+ & \left.\frac{2 k_{2}}{m_{A}}\left(1+\frac{2 m_{A}}{m_{B}} \sin ^{2} \gamma\right)\right]+\frac{2 M k_{1} k_{2}}{m_{B} m_{A}^{2}}=0 .
\end{aligned}
$$

Нормальные колебательные моды малых молекул, как правило, известны. Симметричными нормальными колебаниями являются валентные и деформационные колебания (рис. 3). Их частоты удобно обозначить соответственно $\omega_{v}$ и $\omega_{d}$. Заметим попутно, что приведенные уравнения позволяют решить обратную задачу: по известным частотам нормальных колебаний $\omega_{v}$ и $\omega_{d}$ найти силовые константы $k_{1}$ и $k_{2}$. Результат получается применением теоремы Виета к секулярному уравнению (31):

$$
\begin{aligned}
& \omega_{1}^{2} \equiv \frac{k_{1}}{m_{A}}=\frac{\omega_{v}^{2}+\omega_{d}^{2}+\Omega_{0}^{2}}{2\left(1+\frac{2 m_{A}}{m_{B}} \cos ^{2} \gamma\right)}, \\
& \omega_{2}^{2} \equiv \frac{2 k_{2}}{m_{A}}=\frac{\omega_{v}^{2}+\omega_{d}^{2}-\Omega_{0}^{2}}{2\left(1+\frac{2 m_{A}}{m_{B}} \sin ^{2} \gamma\right)} .
\end{aligned}
$$
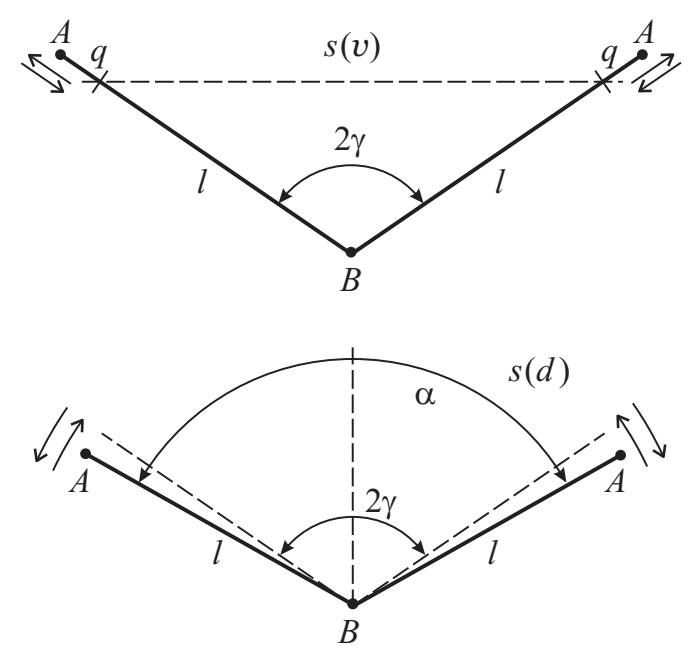

Рис. 3. Симметричные валентное $s(v)$ и деформационное $s(d)$ колебания молекулы $A_{2} B$. 
Здесь

$$
\begin{aligned}
\Omega_{0}^{4} & =\left(\omega_{v}^{2}+\omega_{d}^{2}\right)^{2} \\
& -\frac{4 m_{B}}{M} \omega_{v}^{2} \omega_{d}^{2}\left(1+\frac{2 m_{A}}{m_{B}} \cos ^{2} \gamma\right)\left(1+\frac{2 m_{A}}{m_{B}} \sin ^{2} \gamma\right) .
\end{aligned}
$$

Амплитуды $a_{1}, a_{2}$ связаны друг с другом соотношением

$$
a_{2}=-\frac{m_{B}}{M} \frac{\omega^{2}-\left(\omega_{1}^{2} \sin ^{2} \gamma+\omega_{2}^{2} \cos ^{2} \gamma\right)}{\left(\omega_{2}^{2}-\omega_{1}^{2}\right) \sin \gamma \cos \gamma} a_{1}=b a_{1},
$$

которое следует из первого уравнения системы (30). В частности, для валентных колебаний

$$
b_{v}=-\frac{m_{B}}{M} \frac{\omega_{v}^{2}-\left(\omega_{1}^{2} \sin ^{2} \gamma+\omega_{2}^{2} \cos ^{2} \gamma\right)}{\left(\omega_{2}^{2}-\omega_{1}^{2}\right) \sin \gamma \cos \gamma},
$$

для деформационных

$$
b_{d}=-\frac{m_{B}}{M} \frac{\omega_{d}^{2}-\left(\omega_{1}^{2} \sin ^{2} \gamma+\omega_{2}^{2} \cos ^{2} \gamma\right)}{\left(\omega_{2}^{2}-\omega_{1}^{2}\right) \sin \gamma \cos \gamma} .
$$

Значения величин $\omega_{1,2}$ даются соотношениями (32).

Теперь решение уравнений Лагранжа (28) можно представить в следующем виде:

$$
\begin{gathered}
Q_{s 1}=A_{v} \cos \left(\omega_{v} t+\phi_{v}\right)+A_{d} \cos \left(\omega_{d} t+\phi_{d}\right) \equiv Q_{v}+Q_{d}, \\
Q_{s 2}=b_{v} A_{v} \cos \left(\omega_{v} t+\phi_{v}\right) \\
\quad+b_{d} A_{d} \cos \left(\omega_{d} t+\phi_{d}\right) \equiv b_{v} Q_{v}+b_{d} Q_{d} .
\end{gathered}
$$

Подстановка (35) диагонализует квадратичную форму (27):

$$
\mathscr{L}_{s}=\frac{1}{2} M_{v} \dot{Q}_{v}^{2}+\frac{1}{2} M_{v} \omega_{v}^{2} Q_{v}^{2}+\frac{1}{2} M_{d} \dot{Q}_{d}^{2}+\frac{1}{2} M_{d} \omega_{d}^{2} Q_{d}^{2} .
$$

Здесь приведенные массы, соответствующие нормальным колебаниям $Q_{v, d}$, даются следующими соотношениями:

$$
\begin{gathered}
M_{v}=\frac{1}{2} m_{A}\left(1+b_{v}^{2} \frac{M}{m_{B}}\right), \\
M_{d}=\frac{1}{2} m_{A}\left(1+b_{d}^{2} \frac{M}{m_{B}}\right)
\end{gathered}
$$

(см. также (33), (34)).

Таким образом, в обобщенных координатах $Q_{a}, Q_{v}, Q_{d}$ лагранжиан колебаний симметричной трехатомной нелинейной молекулы (24) соответствует трем независимым линейным гармоническим осцилляторам, колеблющимся с нормальными частотами $\omega_{a}, \omega_{v}, \omega_{d}$. Соответствующие приведенные массы даются соотношениями (26) и (36). Связь нормальных координат с декартовыми дается соотношениями (21) и (35), причем нулевые значения координат соответствуют равновесной конфигурации ядер в молекуле.

Соотношения (21), (26), (35) и (36) решают поставленную задачу согласованного введения соответствующих нормальных координат и приведенных масс в нелинейной молекуле $A_{2} B$. Отметим неоднозначность их выбора, поскольку функция Лагранжа нормального осциллятора не изменяется после масштабирования приведенной массы $M$ и соответствующей нормальной координаты $Q$ :

$$
Q \rightarrow Q / \beta, \quad M \rightarrow \beta^{2} M,
$$

где $\beta$ - произвольная положительная константа.

\section{Изменение колебательных параметров в лазерном поле: молекула $A_{2} B$}

В случае трехатомной нелинейной симметричной молекулы $A_{2} B$ в выражениях (15) и (10) следует положить $k=a, v, d$ (обозначения для колебательных мод см. выше).

Изменение нормальных частот под воздействием внешнего поля дается выражением (15). Обратим внимание на инвариантность уравнения (15) относительно масштабирующего преобразования (37). Если ориентация молекулы относительно вектора линейной поляризации излучения $\boldsymbol{u}$ задается углами Эйлера $(\theta, \phi)$, то

$$
\begin{aligned}
& \alpha_{s s^{\prime}}^{(n)} F_{0 s} F_{0 s^{\prime}} / F_{0}^{2}=\left[\alpha_{x x}^{(n)} \cos ^{2} \phi+\alpha_{y y}^{(n)} \sin ^{2} \phi\right] \sin ^{2} \theta \\
& \quad+\alpha_{z z}^{(n)} \cos ^{2} \theta+2\left\{\left[\alpha_{z y}^{(n)} \sin \phi-\alpha_{z x}^{(n)} \cos \phi\right] \cos \theta\right. \\
& \left.\quad-\alpha_{x y}^{(n)} \sin \theta \cos \theta \sin \phi\right\} \sin \theta,
\end{aligned}
$$

где $\alpha_{s s^{\prime}}^{(n)}$ - декартовы компоненты (собственная система координат молекулы) производной $n$-го порядка тензора поляризуемости $\hat{\alpha}$ по нормальным координатам. Множитель 2 учитывает симметричность тензора $\left(\alpha_{s s^{\prime}}^{(n)}=\alpha_{s^{\prime} s}^{(n)}\right)$.

Напомним способ задания ориентации молекулы с помощью углов Эйлера применительно к нашему случаю молекулы $A_{2} B$. Пусть в исходном положении молекула находится в плоскости $y z$, атом $B-$ в начале координат, вектор $\boldsymbol{u}$ направлен вдоль оси симметрии молекулы (другими словами - вдоль биссектрисы $z$ ). Тогда требуемая ориентация получается в результате двух последовательных поворотов. Вначале молекула поворачивается вокруг оси $y$ против часовой стрелки на угол $0 \leq \theta \leq \pi$ (рис. 4, $a$ ). В результате ось $z$ переходит в $z^{\prime}$. Затем молекула поворачивается вокруг оси $z^{\prime}$ на угол $0 \leq \phi<2 \pi$ (рис. $4, b)$.

Для упрощения анализа начало отсчета нормальных координат молекулы в отсутствие внешних полей удобно выбрать в положении равновесия: $Q_{e a}=Q_{e v}=Q_{e d}=0$.

Из рис. 2 легко увидеть, что вследствие симметрии молекулы $A_{2} B$ относительно биссектрисы валентного угла для антисимметричных колебаний $(a)$

$$
\left.\frac{\partial \hat{\alpha}}{\partial Q_{a}}\right|_{0}=0
$$

Поэтому деформация молекулы $A_{2} B$ в лазерном поле в первом порядке по интенсивности излучения возможна 


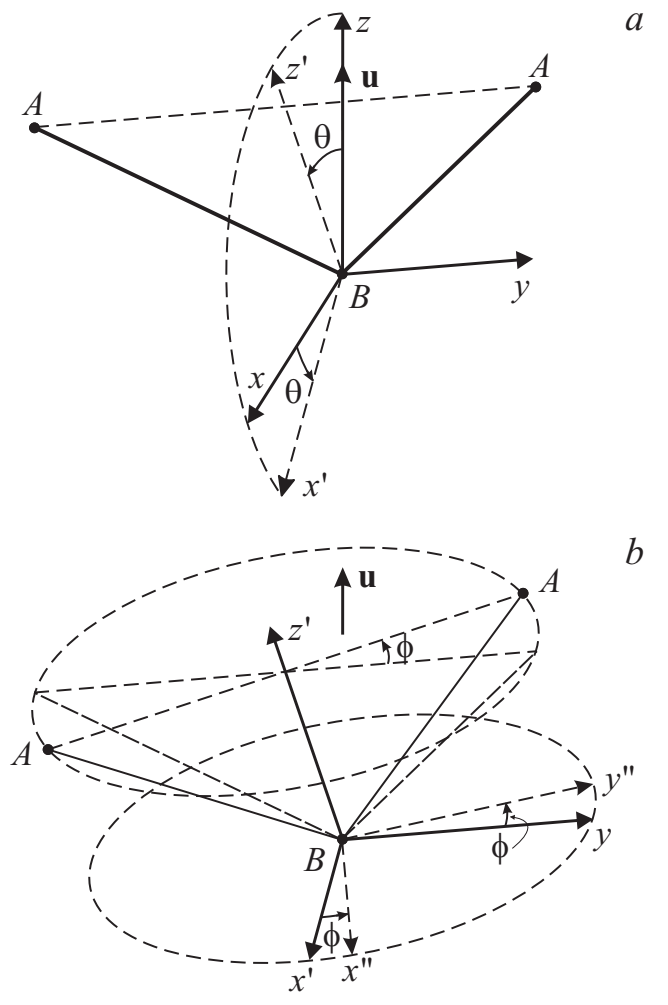

Рис. 4. Задание ориентации молекулы $A_{2} B$ относительно вектора линейной поляризации лазерного излучения $\boldsymbol{u}$ с помощью углов Эйлера $(\theta, \phi)$.

лишь за счет сдвига положений равновесия у симметричных колебаний $(v, d)$ (рис. 3$)$. В частности, изменение валентной длины $A-B$

$$
\begin{aligned}
\Delta l \approx & \frac{1}{2}\left[\left(\sin \gamma+b_{v} \frac{M}{m_{B}} \cos \gamma\right) Q_{E v}\right. \\
& \left.+\left(\sin \gamma+b_{d} \frac{M}{m_{B}} \cos \gamma\right) Q_{E d}\right],
\end{aligned}
$$

изменение валентного угла

$$
\begin{aligned}
\Delta \cos 2 \gamma \approx & -l^{-1} \sin 2 \gamma\left[\left(\cos \gamma-b_{v} \frac{M}{m_{B}} \sin \gamma\right) Q_{E v}\right. \\
& \left.+\left(\cos \gamma-b_{d} \frac{M}{m_{B}} \sin \gamma\right) Q_{E d}\right] .
\end{aligned}
$$

Здесь значения $Q_{E v}$ и $Q_{E d}$ даются формулой (10). Формулы (39), (40) получены из формул (21), (35) в приближении малых отклонений.

\section{Особенности расчетной схемы}

В качестве примеров рассмотрены симметричные нелинейные молекулы ${ }^{1} \mathrm{H}_{2}{ }^{16} \mathrm{O},{ }^{32} \mathrm{~S}^{16} \mathrm{O}_{2}$ и их ионы. Частоты нормальных колебаний молекул даны, например, в NIST Computational Chemistry Comparison and Benchmark DataBase [45]. Недостающие значения колебательных параметров могут быть вычислены с помощью пакета GAUSSIAN. Авторы работы [35] в исследовании молекулы $\mathrm{CO}_{2}$ предлагают использовать для этой цели многоконфигурационные методы c Pople’s базисным набором 6-311G(d).

В настоящей работе для молекулы $\mathrm{H}_{2} \mathrm{O}$ и ее ионов нами использован метод квадратичного взаимодействия конфигураций QCISD [46] с эквивалентным [47] Dunning's базисным набором cc-pVQZ, который в отличие от Pople's наборов пригоден и для вычисления поляризуемостей. Dunning's базисные наборы cc-pVXZ являются корреляционно-согласованнымиполяризационными X-кратно валентно-расщепленными. Существующие наборы могут включать $\mathrm{X}=\mathrm{D}=2, \mathrm{X}=\mathrm{T}=3, \mathrm{X}=\mathrm{Q}=4$, $\mathrm{X}=5$, а также для атомов из начала таблицы Менделеева, $\mathrm{X}=6$. Как показано в [47], удовлетворительная сходимость результатов достигается при $\mathrm{X}=\mathrm{Q}=4$. Для молекул $\mathrm{SO}_{2}$ данный базисный набор оказывается непригодным по причинам, указанным ниже, и мы использовали 6-311G(3df, 3pd).

В табл. 1 даны колебательные параметры рассматриваемых молекул и ионов.

Одним из недостатков метода гауссовых орбиталей является существенное (до 15\%) отличие вычисляемых колебательных частот в молекулах от экспериментальных значений. Для устранения данного недостатка в работе [48] построены таблицы масштабирующих коэффициентов для колебательных частот, получаемых с помощью каждого метода и базисного набора. Однако в этой работе не исследованы корреляционно-согласованные базисные наборы. Вычисленные значения масштабирующих множителей являются результатом подгонки к различным молекулам методом наименьших квадратов и игнорируют индивидуальные свойства атомов и молекул. В настоящей работе мы вычисляли частоты колебаний нейтральных молекул $\mathrm{H}_{2} \mathrm{O}$, затем определяли масштабирующий множитель $f_{\text {sc }}$ для каждой молекулы сравнением с экспериментальными данными [45]. Наконец, вычислялись неизвестные из эксперимента частоты в

Таблица 1. Частоты нормальных колебаний, валентные длины $l$ и углы $2 \gamma$ в симметричных нелинейных трехатомных молекулах и их ионах

\begin{tabular}{l|c|c|c|c|c}
\hline Молекула & $\omega_{a}, \mathrm{~cm}^{-1}$ & $\omega_{v}, \mathrm{~cm}^{-1}$ & $\omega_{d}, \mathrm{~cm}^{-1}$ & $l, \AA$ & $2 \gamma$ \\
\hline $\mathrm{H}_{2} \mathrm{O}^{1}$ & 3942.5 & 3832.2 & 1648.5 & 0.958 & $104.4776^{\circ}$ \\
$\mathrm{H}_{2} \mathrm{O}^{2}$ & 3984.4 & 3879.8 & 1670.4 & 0.956 & $104.3637^{\circ}$ \\
$\mathrm{H}_{2} \mathrm{O}^{+b}$ & 3472.7 & 3418.7 & 1483.7 & 0.997 & $109.4566^{\circ}$ \\
$\mathrm{H}_{2} \mathrm{O}^{2+b}$ & 1862.2 & 1462.3 & 815.6 & 1.177 & $125.2367^{\circ}$ \\
$\mathrm{SO}_{2}{ }^{a}$ & 1380.91 & 1167.6 & 526.27 & 1.432 & $119.5^{\circ}$ \\
$\mathrm{SO}_{2}{ }^{b}$ & 1409.76 & 1209.3 & 536.59 & 1.432 & $118.8225^{\circ}$ \\
$\mathrm{SO}_{2}{ }^{+a}$ & 1227.73 & 1074.3 & 409.15 & - & - \\
$\mathrm{SO}_{2}{ }^{+b}$ & 1253.38 & 1112.7 & 417.17 & 1.423 & $131.9789^{\circ}$
\end{tabular}

${ }^{1}$ Взято из [45].

${ }^{2}$ Рассчитано в пакете GAUSSIAN с базисным набором QCISD/cc-pVQZ для $\mathrm{H}_{2} \mathrm{O}$ и QCISD/6-311G(3df,3pd) для $\mathrm{SO}_{2}$. 
Таблица 2. Компоненты тензора поляризуемости $\hat{\alpha}$ (в ат. ед., $\left.a_{\mathrm{B}}^{3}\right)$ в главных осях и вектора постоянного дипольного момента $\boldsymbol{p}(\mathrm{D})$ в симметричных нелинейных трехатомных молекулах и их ионах, рассчитанные в пакете GAUSSIAN с помощью QCISD/cc-pVQZ (для $\mathrm{H}_{2} \mathrm{O}$ ) и QCISD/6-311G(3df,3pd) (для $\mathrm{SO}_{2}$ ). Ориентация молекулы указана на рис. 1 (ось $z$ направлена вдоль биссектрисы валентного угла, ось $x$ направлена перпендикулярно плоскости молекулы). Начало координат помещено в центр заряда ядер. Экспериментальные значения взяты из [45]

\begin{tabular}{l|c|c|c|c|c}
\hline Величина & $\mathrm{H}_{2} \mathrm{O}$ & $\mathrm{H}_{2} \mathrm{O}^{+}$ & $\mathrm{H}_{2} \mathrm{O}^{2+}$ & $\mathrm{SO}_{2}$ & $\mathrm{SO}_{2}^{+}$ \\
\hline$p_{z}$ & 1.905287 & 2.114637 & 2.927798 & -1.745838 & -1.294614 \\
$p_{z, \exp }$ & 1.855 & - & - & -1.630 & - \\
$\alpha_{x x}$ & 6.605699 & 3.965230 & 2.982851 & 16.896724 & 14.225520 \\
$\alpha_{y y}$ & 8.767866 & 6.184936 & 5.713475 & 31.730373 & 31.972589 \\
$\alpha_{z z}$ & 7.916490 & 4.940379 & 3.914259 & 20.357304 & 17.026385 \\
$\bar{\alpha}$ & 7.763 & 5.030 & 4.204 & 22.995 & 21.075 \\
$\bar{\alpha}_{\text {exр }}$ & 10.128 & - & - & 26.194 & -
\end{tabular}

молекулярных ионах и масштабировались полученными ранее множителями $f_{\text {sc: }}$ :

$$
f_{\mathrm{sc}}^{(a)}=0.9895, \quad f_{\mathrm{sc}}^{(v)}=0.9877, \quad f_{\mathrm{sc}}^{(d)}=0.9869,
$$

что несколько лучше по сравнению с усредненным значением

$$
f_{\text {sc }}[\text { QCISD-fc/6-31G(d) }]=0.9537,
$$

полученным в работе [48]. Масштабированные таким образом частоты нормальных колебаний ионов даны в табл. 1. Поскольку вычисленные геометрические параметры нейтральных молекул отличаются от экспериментальных значений на величину $\lesssim 0.1 \%$, то для ионов были использованы рассчитанные теми же методами параметры.

Использование многоконфигурационных методов является обязательным в расчетах тензора поляризуемости молекул с незамкнутыми оболочками. Одноконфигурационные методы (например, HF) или методы функционала плотности (например, B3LYP) в этом случае приводят к абсурдным результатам. В частности, в двухатомных молекулах $\left(\mathrm{HCl}^{+}, \mathrm{O}_{2}, \mathrm{O}_{2}^{+}\right.$, $\mathrm{NO}$ ит.д.) для поперечных компонент тензора нарушается осевая симметрия:

$$
\alpha_{x x}=\alpha_{y y}=\alpha_{\perp} .
$$

Более того, отдельные компоненты тензора могут получиться отрицательными. Среди многоконфигурационных методов QCISD представляется наиболее предпочтительным, поскольку, в отличие от MCSCF, он не требует явного указания задействованных конфигураций и обеспечивает точность выполнения условия (41) в пределах $0.01 \%$.

Значение поляризуемости весьма чувствительно к выбору базисного набора. Так, для атома водорода набор cc-pVQZ дает $\alpha=2.737 a_{\mathrm{B}}^{3}$, в то время как 6-311(d) - на $\sim 10 \%$ ниже. Включение диффузных функций в корреляционно-согласованный набор (aug-cc-pVQZ) приводит к поляризуемости $\alpha=4.496 a_{\mathrm{B}}^{3}$, почти совпадающей с точным значением $\left(\alpha=\frac{9}{2} a_{\mathrm{B}}^{3}\right)$. В то же время дополнение валентно-расщепленных Pople's наборов диффузными функциями не изменяет результат заметным образом. В более сложных молекулах расхождение вычисленных поляризуемостей с их экспериментальными значениями оказывается менее драматичным (табл. 2). К сожалению, экспериментальные данные [45] содержат лишь скалярные компоненты поляризуемостей

$$
\bar{\alpha}=\frac{1}{3}\left(\alpha_{x x}+\alpha_{y y}+\alpha_{z z}\right),
$$

что затрудняет сравнение с экспериментом. Расчет с использованием QCISD/aug-cc-pVQZ технически неосуществим в связи с требованием большого объема оперативной памяти. В отличие от поляризуемости постоянный дипольный момент зависит от выбора базисного набора и метода существенно меньше.

Дифференцирование тензора поляризуемости $\hat{\alpha}$ по нормальным координатам, необходимое при использовании формул (10) и (15), выполнялось по пяти точкам с шагом $0.005 \AA$. К сожалению, для молекулы $\mathrm{SO}_{2}$ с базисным набором сc-pVQZ значение $\partial^{2} \hat{\alpha} / \partial Q_{a}^{2}$ оказалось аномально большим ( $\left.\gtrsim 1 \cdot 10^{3} a_{\mathrm{B}}\right)$. В результате при интенсивности лазерного излучения $\sim 1 \cdot 10^{14} \mathrm{~W} / \mathrm{cm}^{2}$ поправка к частоте $\omega_{a}^{2}$ превысила невозмущенное значение в десятки раз. Поскольку физические причины недостаточно гладкой зависимости $\hat{\alpha}\left(Q_{a}\right)$ отсутствуют, следует предположить ограниченность применимости Dunning's корреляционно-согласованных базисных наборов. Поэтому для молекулы $\mathrm{SO}_{2}$ использован Pople's валентно-расщепленный базис 6-311(3df,3pd), содержащий максимально полный для 6-311 набор поляризационных волновых функций.

\section{Результаты вычислений}

Изменения колебательных параметров молекул (нормальных частот и положений равновесия) в монохроматическом линейно поляризованном лазерном поле рассчитываются по формулам (10) и (15). Изменения валентных длин и углов - соответственно по формулам (39) и (40). Использовались статические значения поляризуемостей, которые слабо отличаются 
Таблица 3. Относительные изменения частоты нормальных колебаний $\Delta\left(\omega_{i}\right)=\Delta \omega_{i} / \omega_{i}$, валентных длин $\Delta(l)=\Delta l / l$ и углов $\Delta(\gamma)=\Delta \gamma / \gamma \quad$ (в \%) в симметричных нелинейных трехатомных молекулах и их ионах в монохроматическом линейно поляризованном лазерном поле с интенсивностью $1 \cdot 10^{14} \mathrm{~W} / \mathrm{cm}^{2}$

\begin{tabular}{l|c|c|c|c|c}
\hline Молекула & $\Delta\left(\omega_{a}\right)$ & $\Delta\left(\omega_{v}\right)$ & $\Delta\left(\omega_{d}\right)$ & $\Delta(l)$ & $\Delta(\gamma)$ \\
\hline \multicolumn{6}{c}{$\boldsymbol{u}=\boldsymbol{e}_{x}$} \\
\hline $\mathrm{H}_{2} \mathrm{O}$ & -0.0044 & +0.018 & -0.028 & +0.090 & +0.012 \\
$\mathrm{H}_{2} \mathrm{O}^{+}$ & -2.553 & -0.3742 & -0.8737 & +0.5077 & -0.0372 \\
$\mathrm{H}_{2} \mathrm{O}^{2+a}$ & +1.081 & -0.2773 & -1.157 & +1.734 & -0.1717 \\
$\mathrm{SO}_{2}$ & -0.1020 & -0.1733 & -0.4133 & +0.0560 & +0.1660 \\
$\mathrm{SO}_{2}^{+}$ & -0.2045 & +0.0077 & -0.6855 & +0.1191 & +0.0070 \\
\hline \multicolumn{5}{c}{$\boldsymbol{u}=\boldsymbol{e}_{y}$} \\
\hline $\mathrm{H}_{2} \mathrm{O}$ & -0.37 & -0.73 & -1.33 & +0.61 & +0.53 \\
$\mathrm{H}_{2} \mathrm{O}^{+}$ & -2.332 & -5.195 & -8.947 & +4.820 & +4.640 \\
$\mathrm{H}_{2} \mathrm{O}^{2+a}$ & +5.223 & -18.09 & -11.05 & +27.68 & +8.013 \\
$\mathrm{SO}_{2}$ & -2.689 & -0.6280 & -0.4133 & +0.7295 & +0.8999 \\
$\mathrm{SO}_{2}^{+}$ & -5.112 & -1.438 & -10.88 & -0.2573 & +0.6316 \\
\hline \multicolumn{5}{c}{$\boldsymbol{u}=\boldsymbol{e}_{z}$} \\
\hline $\mathrm{H}_{2} \mathrm{O}$ & -0.26 & -0.26 & -0.87 & +0.37 & +0.012 \\
$\mathrm{H}_{2} \mathrm{O}^{+}$ & -1.266 & -1.870 & -7.529 & +2.439 & -5.802 \\
$\mathrm{H}_{2} \mathrm{O}^{2+a}$ & +1.153 & -5.173 & -10.59 & +10.03 & -12.74 \\
$\mathrm{SO}_{2}$ & -0.66646 & -0.3259 & -1.031 & +0.1751 & -0.6804 \\
$\mathrm{SO}_{2}^{+}$ & -0.2364 & +0.4528 & -0.8767 & +0.1609 & -0.9900
\end{tabular}

а Для интенсивности $1 \cdot 10^{15} \mathrm{~W} / \mathrm{cm}^{2}$.

от динамических в ближнем ИК диапазоне излучения. Результаты расчетов по формулам (15), (39) и (40) при интенсивности излучения $1 \cdot 10^{14} \mathrm{~W} / \mathrm{cm}^{2}$ и различных направлениях вектора поляризации $\boldsymbol{u}$ даны в табл. 3 для молекул $\mathrm{H}_{2} \mathrm{O}, \mathrm{H}_{2} \mathrm{O}^{+}$и $\mathrm{SO}_{2}$. Использовалась программа GAUSSIAN c QCISD/cc-pVQZ. Из таблицы видно, что изменение параметров колебаний оказывается наиболее существенным, если вектор поляризации лежит в плоскости молекулы. Также можно видеть, что если вектор поляризации параллелен оси симметрии молекулы, то ее излом под воздействием лазерного поля становится более заметным (валентный угол уменьшается). Другие направления вектора поляризации, как правило, приводят к „выпрямлению“ молекулы.

Учет изменения геометрических параметров и частот колебаний, изложенный в настоящей работе, приводит к изменению вероятности туннельной ионизации молекулы $\mathrm{SO}_{2}$ в лазерном поле с интенсивностью $\sim 1 \cdot 10^{14} \mathrm{~W} / \mathrm{cm}^{2}$ до 20 раз в зависимости от ориентации, тогда как для молекулы $\mathrm{H}_{2} \mathrm{O}$ изменение не превышает нескольких процентов. Причина такого различия связана с тем, что, несмотря на геометрическое сходство, молекулы $\mathrm{H}_{2} \mathrm{O}$ и $\mathrm{SO}_{2}$ имеют принципиально различную электронную структуру.

Во-первых, в молекуле $\mathrm{H}_{2} \mathrm{O}$ все орбитали имеют нечетные значения проекции орбитального момента на ось симметрии, в то время как в молекуле $\mathrm{SO}_{2}$ наоборот все орбитали имеют четные значения проекции момента. Это означает, что во всех орбиталях молекулы воды отсутствует вклад в ионизацию $\sigma$-состояний. Поэтому вероятность туннелирования электрона из молекулы воды подавлена при параллельной ориентации оси симметрии и электрического вектора, когда влияние постоянного дипольного момента максимально. При перпендикулярной ориентации вероятность туннелирования из молекулы $\mathrm{H}_{2} \mathrm{O}$ максимальна и практически на подвержена влиянию постоянного дипольного момента.

Во-вторых, вследствие высокой электроотрицательности кислорода в обеих молекулах на атомах кислорода имеется избыточный отрицательный Mulliken's заряд. Поэтому в этих молекулах постоянные дипольные моменты $\boldsymbol{p}_{i}$ имеют противоположные направления. В молекуле воды вектор постоянного дипольного момента направлен от атома О к середине отрезка $\mathrm{H}-\mathrm{H}$. В молекуле диоксида серы наоборот: вектор постоянного дипольного момента направлен от середины отрезка $\mathrm{O}-\mathrm{O}$ к атому $\mathrm{S}$ (табл. 2).

Как видно из табл. 3, изменение частот наиболее существенно при перпендикулярной ориентации $\boldsymbol{p}_{i}$ и $\boldsymbol{u}$, что приводит к значительному монотонному изменению факторов Франка-Кондона с ростом угла между $\boldsymbol{p}_{i}$ и $\boldsymbol{u}$. Вследствие этого изменяется и вероятность туннелирования. Однако по причине вышеупомянутых различий в электронной структуре с ростом угла факторы ФранкаКондона в молекуле $\mathrm{H}_{2} \mathrm{O}$ возрастают, а в молекуле $\mathrm{SO}_{2}$ убывают. Влияние постоянного дипольного момента на туннельную ионизацию учитывается дополнительным множителем, который не влияет на отношение вероятностей туннелирования с учетом деформации молекулы в лазерном поле и без ее учета.

Работа была выполнена при поддержке Министерства образования и науки РФ в рамках Госзадания № 3.1761.2017/4.6 с использованием высокопроизводительного компьютерного кластера Воронежского государственного университета.

\section{Список литературы}

[1] Alnaser A.S., Kübel M., Siemering R., Bergues B. et al. // Nat. Commun. 2014. V. 5. P. 3800. doi $10.1038 /$ ncomms 4800

[2] Kübel M., Alnaser A.S., Bergues B., Pischke T. et al. // New J. Phys. 2014. V. 16. N 6. P. 065017. doi 10.1088/1367-2630/16/6/065017

[3] Li H., Mignolet B., Wachter G., Skruszewicz S. et al. // Phys. Rev. Lett. 2015. V. 114. P. 123004. doi 10.1103/PhysRevLett.114.123004

[4] Usenko S., Schüler M., Azima A., Jakob M. et al. // New J. Phys. 2016. V. 18. N 11. P. 113055. doi 10.1088/1367-2630/18/11/113055

[5] Steinitz U., Prior Y., Averbukh I.S. // Phys. Rev. Lett. 2014. V. 112. P. 013004. doi 10.1103/PhysRevLett.112.013004

[6] Reichert M., Zhao P., Reed J.M., Ensley T.R. et al. // Opt. Express. 2015. V. 23. N 17. P. 22224-22237. doi 10.1364/OE.23.022224 
[7] Mizuse K., Kitano K., Hasegawa H., Ohshima Y. // Sci. Adv. 2015. V. 1. N 6. P. e1400185. doi 10.1126/sciadv. 1400185

[8] Korobenko A., Hepburn J.W., Milner V. // Phys. Chem. Chem. Phys. 2015. V. 17. P. 951-956. doi 10.1039/C4CP04434G

[9] Тен Г.Н., Бурова Т.Г., Березин К.В., Яковлева А.А. u др. // Опт. и спектр. 2012. Т. 113. В. 5. С 539-547. doi 10.1134/S0030400X12090160

[10] Бункин С., Гладков С., Морозов В. // Опт. и спектр. 1987. T. 62. B. 2. C. $356-359$.

[11] Тулуб А., Симон К. // Опт. и спектр. 2007. Т. 102. В. 2. C. 219-226. doi 10.1134/S0030400X07020051

[12] Albeck Y., Lerner G., Kandhasamy D.M., Chandrasekaran V., Strasser D. // Phys. Rev. A. 2015. V. 92. P. 061401. doi 10.1103/PhysRevA.92.061401

[13] Kandhasamy D.M., Albeck Y., Jagtap K., Strasser D. // J. Phys. Chem. A. 2015. V. 119. N 29. P. 8076-8082. doi 10.1021/acs.jpca.5b04101

[14] Albeck Y., Lerner G., Kandhasamy D.M., Chandrasekaran V., Strasser D. // J. Phys. Chem. A. 2016. V. 120. N 19. P. 3246-3252. doi 10.1021/acs.jpca.5b11792

[15] Morimoto Y., Kanya R., Yamanouchi K. // Phys. Rev. Lett. 2015. V. 115. P. 123201. doi 10.1103/PhysRevLett.115.123201

[16] Зон Б.А. // ЖЭТФ. 1977. Т. 73. С. 128-133.

[17] Kern C.W., Matcha R.L. // J. Chem. Phys. 1968. V. 49. N 5. P. 2081-2091. doi 10.1063/1.1670369

[18] Pandey P.K.K., Santry D.P. // J. Chem. Phys. 1980. V. 73. N 6. P. 2899-2901. doi $10.1063 / 1.440460$

[19] Зон Б.А., Шолохов Е.И.// ЖЭТФ. 1976. Т. 70. С. 887-898.

[20] Bishop D.M., Solunac S.A. // Phys. Rev. Lett. 1985. V. 55. P. 1986-1988. doi 10.1103/PhysRevLett.55.1986

[21] Bishop D.M. // J. Chem. Phys. 1993. V. 98. N 4. P. 3179-3184. doi 10.1063/1.464090

[22] Bishop D.M. //Rev. Mod. Phys. 1990. V. 62. P. 343-374. doi 10.1103/RevModPhys.62.343

[23] Zon B.A. // Chem. Phys. Lett. 1996. V. 262. N 6. P. 744-746. doi 10.1016/S00092614(96)01155-4

[24] Kornev A.S., Zon B.A. // Phys. Rev. A. 2012. V. 86. P. 043401. doi 10.1103/PhysRevA.86.043401

[25] Kornev A., Zon B. // Laser Phys. 2014. V. 24. N 11. P. 115302. doi $10.1088 / 1054660 \mathrm{X} / 24 / 11 / 115302$

[26] Kornev A.S., Zon B.A. // Phys. Rev. A. 2015. V. 92. P. 033420. doi 10.1103/PhysRevA.92.033420

[27] Kornev A.S., Semiletov I.M., Zon B.A. // Laser Phys. 2016. V. 26. N 5. P. 055302.

[28] Küpper J., Stern S., Holmegaard L., Filsinger F. et al. // Phys. Rev. Lett. 2014. V. 112. P. 083002. doi 10.1103/PhysRevLett.112.083002

[29] Ren X., Makhija V., Kumarappan V. // Phys. Rev. Lett. 2014. V. 112. P. 173602. doi 10.1103/PhysRevLett.112.173602

[30] Pullen M.G., Wolter B., Le A.-T., Baudisch M. et al. // Nat. Commun. 2015. V. 6. P. 7262. doi 10.1038/ncomms 8262

[31] Song Q., Li Z., Li H., Lu P. et al. // Opt. Express. 2017. V. 25. N 3. P. 2221-2227. doi 10.1364/OE.25.002221

[32] Hishikawa A., Iwamae A., Yamanouchi K. // J. Chem. Phys. 1999. V. 111. N 19. P. 8871-8878. doi 10.1063/1.480233

[33] Sanderson J.H., El-Zein A., Bryan W.A., Newell W.R. et al. // Phys. Rev. A. 1999. V. 59. P. R2567-R2570. doi 10.1103/PhysRevA.59.R2567

[34] Bryan W.A., Sanderson J.H., El-Zein A., Newell W.R. et al. // J. Phys. B: At. Mol. Opt. Phys. 2000. V. 33. N 4. P. 745-766. doi 10.1088/0953-4075/33/4/312
[35] Kono H., Koseki S., Shiota M., Fujimura Y. // J. Phys. Chem. A. 2001. V. 105. N 23. P. 5627-5636. doi 10.1021/jp003848i

[36] Comstock M., Senekerimyan V., Dantus M. // J. Phys. Chem. A. 2003. V. 107. N 40. P. 8271-8281. doi 10.1021/jp0278115

[37] Hishikawa A., Hasegawa H., Yamanouchi K. // Chem. Phys. Lett. 2004. V. 388. N 1-3. P. 1-6. doi 10.1016/j.cplett.2004.01.128

[38] Hirohiko K., Yukio S., Manabu K., Katsunori N., Tsuyoshi K. // Bull. Chem. Soc. Jpn. 2006. V. 79. N 2. P. 196-227. doi 10.1246/bcsj.79.196

[39] Chen G.-Y., Wang Z.W., Hill W.T. // Phys. Rev. A. 2009. V. 79. P. 011401. doi 10.1103/PhysRevA.79.011401

[40] Wells E., Rallis C., Zohrabi M., Siemering R. et al. // Nat. Commun. 2013. V. 4. P. 2895. doi 10.1038/ncomms 3895

[41] Герцберг Г. Колебательные и вращательные спектры многоатомных молекул. М.: Иностранная литература, 1949. $413 \mathrm{c}$.

[42] Chernov V.E., Kornev A.S., Zon B.A. // Laser Phys. Lett. 2017. V. 14.

[43] Герцберг Г. Электронные спектры и строение многоатомных молекул. М.: Мир, 1969. 772 с.

[44] Волькенштейн М.В., Грибов Л.А., Ельяшевич М.А., Степанов Б.И. Колебания молекул. М.: Наука, 1972. 700 с.

[45] NIST computational chemistry comparison and benchmark database (release18) [Electronic resource]// Ed. by Russell D. Johnson III. URL: http://cccbdb.nist.gov (online; accessed: October 2016)

[46] Pople J., Head-Gordon M. // J. Chem. Phys. 1987. V. 87. N 10. P. 5968-5975. doi $10.1063 / 1.453520$

[47] Sherill C.D. Basis sets in quantum chemistry [Electronic resource] / Sherill C.D. URL: http://vergil.chemistry.gatech.edu/ courses/chem6485/pdf/basis-sets.pdf (online; accessed: August 2017)

[48] Scott A., Radom L. // J. Phys. Chem. 1996. V. 100. N 41. P. 16502-16513. doi 10.1021/jp960976r 